\title{
Concluding Address
}

\section{Franco Giovannelli* ${ }^{* \dagger}$}

INAF - Istituto di Astrofisica e Planetologia Spaziali, Via del Fosso del Cavaliere, 100, 00133

Roma, Italy

E-mail: franco.giovannellidiaps.inaf.it

Before I officially conclude this workshop - and far be it from me to attempt more conclusive remarks than those effected by Dmitry Bisikalo, Peter L. Biermann, James H. Beall, and Janusz Ziółkowski - I would like to comment on a few highlights coming from our very fruitful week of discussions about Multifrequency Behaviour of High Energy Cosmic Sources. I make no pretension of completeness in these brief remarks.

XI Multifrequency Behaviour of High Energy Cosmic Sources Workshop 25-30 May 2015

Palermo, Italy

* Speaker.

${ }^{\dagger}$ A footnote may follow. 
Without doubt, the advent of spacecraft and satellite observatories has given a strong impulse to astronomy. Since the mid-seventies, almost all of the electromagnetic spectrum has been continuously surveyed by many space experiments. In addition, Astroparticle Physics, the new field of physics, was born roughly twenty-five years ago from the joining of efforts of the communities of High Energy Astrophysicists and Particle Physicists. During this relatively short time, Astroparticle Physics developed dynamically through the study of cosmic sources that are emitters of photons, charged particles, and neutrinos. Some of these sources can produce gravitational waves which are likely to be detectable in the near future with the new generation of ground, and space-based gravitational wave experiments (for example, LIGO and LISA, respectively) Meanwhile, the Large Hadron Collider (LHC) has produced excellent results related to research on the Higgs boson, and, more broadly, about the study of pp collisions at $\mathrm{TeV}$ energies that have never before been obtained in ground-based laboratories.

These results, together with those coming from the Hubble Space Telescope (HST) with its deep survey of distant objects in the Universe, and with the VHE emission detected from a number of cosmic sources, both galactic and extragalactic, bear witness to the validity of the Big Bang theory described by the standard model. Further, workers analyzing the results from the BCEP2 experiment claim the detection of E-mode and B-mode polarization of the Cosmic Microwave Background (CMB). If B-mode polarization can be confirmed, it would lend credene to the inflationary model of the Universe. However, big discoveries need big confirmations.

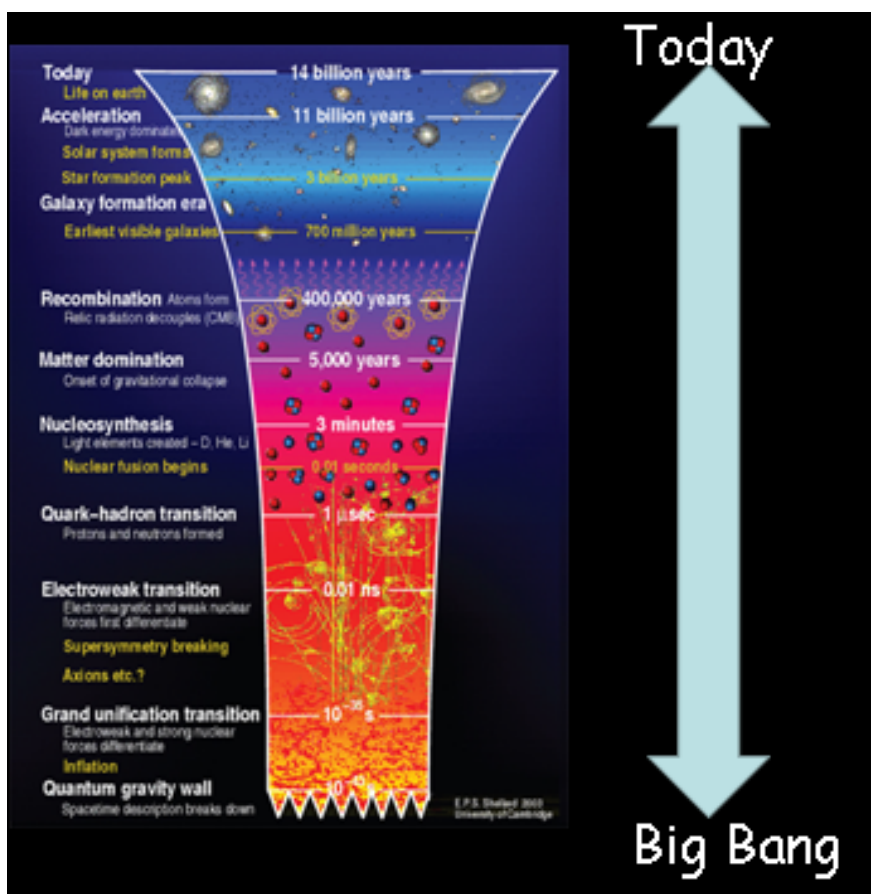

Figure 1: Sketch of the evolution of our Universe from the Big Bang till now (Giovannelli, 2015, after Shellard, E.P.S., 2003).

In this fruitful workshop, we have discussed many topics that can be placed at different stations in the evolutionary track of our Universe, as sketched in Fig. 1 (Giovannelli, 2015, after Shellard, E.P.S., 2003). In other words, we have gone along the bridge of ideas that links the Big Bang and 
Biology.

Among the many experimental and theoretical results of frontier astrophysics discussed during this workshop, I would like to remark on a few of them that, in my opinion, will condition future investigations.

Theoretical and observational works show that jets from AGN can trigger star formation, a phenomenon that has also been found recenly in the surroundings of the microquasar GRS 1915+105. Additional observations of jet-induced star formation by microquasars will be crucial in the next epoch of observations: high-energy and low-energy phenomena are clearly interconnected. In fact, jets from stellar black holes may have been important sources that trigger star formation during the re-ionization epoch of the universe.

There is now rather good agreement about the epoch of re-ionization, as shown in Figure 2 (Giovannelli \& Sabau-Graziati, this workshop). But how re-ionization really occurs is still a subject of debate. Indeed, Dopita et al. (2011) in considering recent observations, show that the measured rates of star formation in the early universe are insufficient to produce re-ionization. Those authors suggest the presence of another source of ionizing photons, perhaps fast accretion shocks formed around the cores of the most massive haloes.

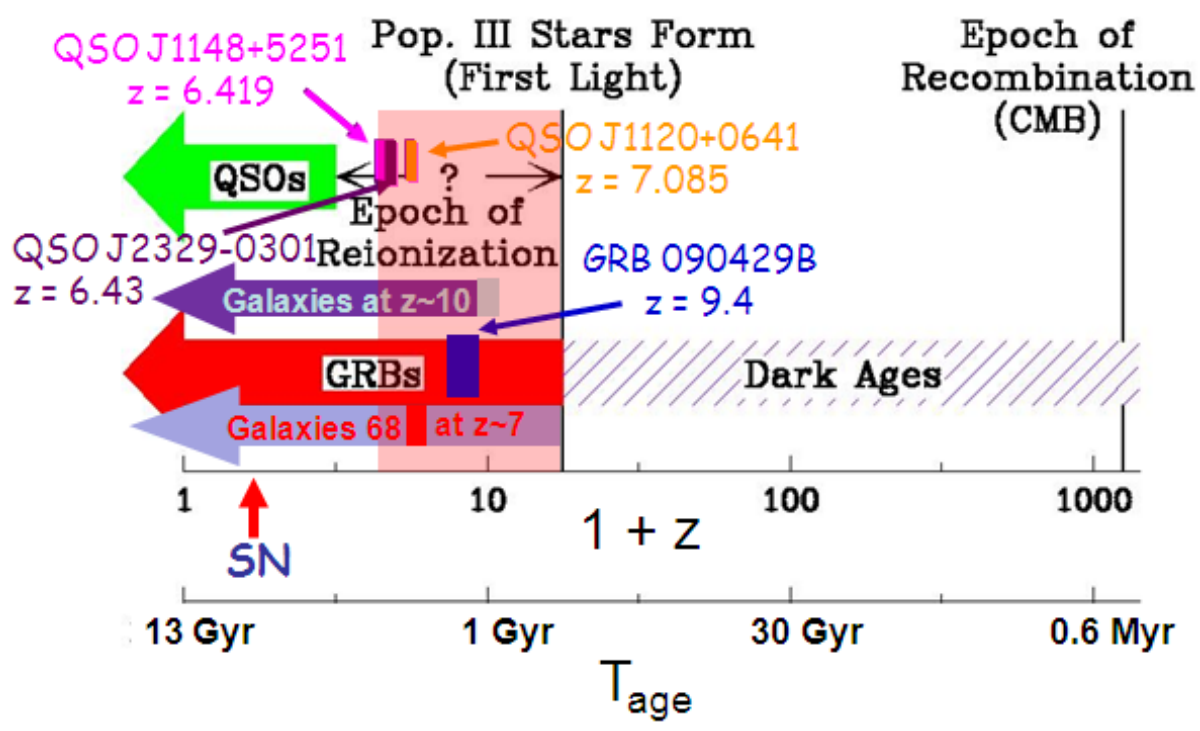

Figure 2: A sketch of reionization epoch (Giovannelli \& Sabau-Graziati, 2015 - after Xiangping Wu's Talk at the Summer School on "Cosmic Reionization" at the KIAA-PKU, Beijing, China, July 1-11, 2008 ).

In another vein, the idea that some GRBs could be associated with the emission of gravitational waves (GWs) is now popular. Indeed, short GRBs are believed to be produced by the mergers of double NSs, double BHs, or NS-BHs in binary systems, and the recent observation of a kilonova associated with GRB130603B (see, e.g. Tanvir et al., 2013) lends support to this hypothesis. Such compact binary coalescences generate strong GWs in the sensitive frequency band of Earth-based gravitational wave detectors. However, till now the sensitivity of GW detectors is not sufficient for such detections. This will be one of the most intriguing investigation of the next few decades.

During this very productive workshop, I believe that we have again demonstrated once more 
the "Vulcano Theorem" enunciated in 1984 in my concluding address: It is possible to develop science seriously even if smiling.

But, as you probably noticed, this workshop has been organized under peaceful and friendly surroundings. Therefore, I would like to mention and to support Tolstoy's philosophy.

"Think again", was written by Lev Nikolàevic Tolstòj (Leo Nikolayevich Tolstoy) in 1904, at the beginning of the Russian-Japanese War. The human condition described by Tolstoy in the state of war is "Like spiders in a glass."

It is a pamphlet that is not only against carnage, but states that

"Again the suffering that benefits nobody, again the lies, and again the universal process of stupidity, the turning of men into beasts...",

Tolstoy also argues against the racism and the hypocrisy of the educated, when he says that

"Scholars ... deal extensively with the laws of the migration of peoples, the relationship between the white and the yellow race, between Buddhism and Christianity, and according to their deductions and considerations justify the killing of men ...".

Mining a similar vein to that of Tolstoy, Gandhi's non-violence doctrine opines that:

"I cannot act in any other way than God requires of me, and therefore, as a man, I cannot take part in any war, neither directly nor through a third party, nor by giving orders, nor by cooperating in any form, nor by encouraging doing it: I cannot, I will not do it".

And finally, I would like to conclude with few wonderful words of Dr Daisaku Ikeda (2001), president of the Soka Gakkai International (SGI), and reported in the booklet entitled For Today and Tomorrow as the thought of the 30th of May:

"The one who has many friends has greater opportunities for growth. In this way, one both makes society a better place, and lives happier and more satisfied. In all cases, human relations, the inter-personal interaction and communication are of vital importance. We must establish and nurture friendship and contacts with many people, both in our environment, and in society in general. In this manner our life will open up and will flourish".

We could go back to early childhood when we were as the "Little Prince", who says that

One sees clearly only with the heart. What is essential is invisible to the eye (from The Little Prince by Antoine de Saint Exupéry, 1943).

The search for the essential is of extreme interest to a large number of men of great learning. These are in agreement with Paul Salahuddin Armstrong, who said in his 2014 talk "Human Family; Past, Present and Future", at the "New Humanity Movement-Event" (Paul Salahuddin Armstrong, 2014):

Today we travel the world, making connections, doing business, and building relationships in person or online with fellow members of our Human Family from all parts of the Earth. We are becoming more conscious that what happens in one place affects people everywhere. We are not alone... We are not isolated... Only through building bridges of Love and Understanding can we ensure the well-being of everyone in our Human Family.

The search for the essential is so important that even famous, noble-minded scientists try to attempt the difficult way of the possible convergence of science and life in its more sublime meaning. For instance, Pier Luigi Luisi founded in 1985 the International Week of Cortona "Science and the Wholeness of Life", dedicated to the integration of Scientific Disciplines and Humanities. Later he published the book "The emergence of Life. From Chemical Origins to Synthetic Biology (Luisi, 
2006) in which he resumed the consecutive stages from prebiotic chemistry to synthetic biology, uniquely combining both approaches. Indeed, the origin of life from inanimate matter has been the focus of much research for decades, both experimentally and philosophically. Friedrich Rolle, a German philosopher and biologist, wrote "The general reasons for this assumption are so categorical that I have no doubt that sooner or later it will be possible to demonstrate such an assumption in an unambiguous and scientific way, or even repeat the process experimentally (Rolle, 1863).

In the book "The Systems View of Life: A Unifying Vision" (Capra \& Luisi, 2014) the authors integrate in a single framework of coherent thought the ideas, models and theories that are the foundation of the systemic vision of life, highlighting its the economic, ecological, political and spiritual implications.

Personally, I would like to reiterate and underscore some fundamental concepts in the book which I completely share.

Life is a network of complex and inseparable relationships that renders the understanding of an individual phenomenon indivisible from the understanding the entire ecosystem in which it occurs. Therefore the answers can not be found by relying exclusively to the scientific method. A "holistic" approach is now required, one that is able to reflect on connectivity, relationships and contexts as well as properties and functions of the individual parts.

The discipline that best reflects the systemic vision of life is ecology, which reconnects the life sciences with the earth sciences and studies the interaction of organisms with each other and with the surrounding environment. The new ecological science - that has emerged from organismic biology only in the late twentieth century, when the concept of ecosystem developed - is not anthropocentric but eco-centric, that is characterized by the awareness that all living things are tied together in networks of interdependence.

Ecology is the ideal bridge between science and spirituality. In fact, within the systemic view of life, it is essential the concept of balance between science - responsible for the material and technological progress - and spirituality, responsible for the internal growth of individuals and ethical limitations imposed by the excessive consumption of resources of the planet. The balance between science and spirituality determines the welfare of society.

The bridge between the Big Bang and Biology ferries us from the original point to the biologically active side where sentient life and then science start. But to close correctly the "run" of it, it is necessary to cross one bridge more: The bridge between science and spirituality. If this bridge is properly covered, our society will flourish.

Personally, I feel obligated to point out some observations that seem fundamental about the philosophical and social implications of contemporary science. These observations lead to interesting conclusions about the origin of life and self-organization of natural and synthetic systems. These findings are in keeping with the Buddhist view of the universe. It is understood as a living organism being composed of myriads of components all related and interacting with one another. Life can be seen as a system of interconnected autopoietic systems. The organism interacts with the environment in a "cognitive" way. At the same time, the organism "creates" its own environment and the environment allows the creation of the organism. But this is the concept of dependent origin. According to this concept, every phenomenon is the product of the interaction of every other phenomenon in the universe. 
The consequence of this view are of extraordinary importance, above all in ethics: it asserts that all living beings and their environment are inextricably linked, and that their essence is not absolute but "of relationship." It leads us to respect every individual being and its inherent rights. In other words, this view leads us to live and act without distinguishing our own happiness from that of others. Ultimately this view leads to the TOTAL RESPECT OF LIFE in the most general meaning.

It is my pleasure to thank:

- the Scientific Organizing Committee (James H. Beall, Dmitry Bisikalo, - Thomas Boller, Franco Giovannelli - (Chairperson), René Hudeč, Lola Sabau-Graziati, and Andrea Santangelo);

- The super-efficient Local Organizing Committe (Franco Giovannelli - (Chairperson), Daniela Giovannini, Paolo Persi, and Francesco Reale - (Co-Chairperson).

Particular warm thanks to:

- ALL SPEAKERS, who rendered this workshop very interesting

- ALL PARTICIPANTS, who breathed life into the workshop with their question-answers, and for the friendly atmosphere they provided

- The FOUR COLLEAGUES and FRIENDS (Dmitry, Peter, Jim \& Janusz) who kindly accepted the not so easy task of making the concluding remarks of the workshop.

- The student of the second class of INDA directed by Flavia Giovannelli for the splendid Performance Bacco in Toscana.

- To all the staff of the SPLENDID Hotel La TORRE.

- Finally, on behalf of all participants, I would like to express my warm tanks to the Chèf, Mr Daniele Inzerillo, who prepared for us a large number of delicacies.

A special grateful thanks goes to:

- The Informatic Operator (Francesco Reale (ISC/CNR), alias FIGARO, the factotum) for helping in informatics and solving all related problems during the preparation and development of the workshop and in Mondello, at the registration desk, in the conference room, on the terrace, and ... more;

- The Executive Secretary Daniela Giovannini (IBCN/CNR), who smoothed all the inconveniences that occurred at the registration desk with professionalism and moreover with her nice smile;

Without the presence of Francesco and Daniela it would not have been possible to organize the workshop!

I hope to meet all of you once again during our next Frascati Workshop. 


\section{References}

[1] Capra, F. \& Luisi, P.L.: 2014, The Systems View of Life: A Unifying Vision, Cambridge University Press.

[2] de Saint Exupéry, Antoine: 1943, Le Petit Prince, Gallimard, France. English version The Little Prince, Reynal \& Hitchcock, U.S.A.

[3] Dopita, M.A.: 2011, Astrophys. Space Sci., 335, 345-352.

[4] Giovannelli, F.: 2015, talk at the Frascati Workshop 2015 on Multifrequency Behaviour of High Energy Cosmic Sources - XI.

[5] Giovannelli, F. \& Sabau-Graziati, L.: 2015, These proceedings.

[6] Ikeda, D.: 2001, For Today and Tomorrow - The thought of 30th May, Edizioni Esperia, Italy.

[7] Luisi, P.L.: 2006, The Emergence of Life. From Chemical Origin to Synthetic Biology, Cambridge Univeristy Press.

[8] Rolle, F.: 1863, Ch.Darwin's Lehre von der Entstehung der Arten, in J.C. Hermann, Ihrer Anwendung auf die Schöpfunggeschichte.

[9] Salahuddin Armstrong, P.: 2014, April 13, Speech originally delivered at the New Humanity Movement event.

[10] Tanvir, N.R. et al.: 2013, Nature, 500, 547.

[11] Tolstoy, L.: 1904, Think Again, Pamphlet published in the Times of London on 27th June 1904.

[12] Wu, Xiangping: 2008, Talk at the Summer School on "Cosmic Reionization" at the KIAA- PKU, Beijing, China, July 1-11. 